\title{
Radiological features of sacrococcygeal teratomas in fetal magnetic resonance imaging and computed tomography - a case report
}

\author{
Oliver P. Firszt ${ }^{1 A, B, C, D, E, F}$, Jolanta Myga-Porosiło ${ }^{2 A, B, D, E, F}$, Katarzyna Pośpieszny ${ }^{2 A, B}$, Tomasz Golus ${ }^{2 B, C, D}$, \\ Sylwia Trzeszkowska-Rotkegel ${ }^{2 B, D}$, Jan Głowacki ${ }^{2 B, E}$, Wojciech Sraga ${ }^{2 B, D}$, Ewa Kluczewska ${ }^{2 D, E, F}$ \\ 'Student Research Group, Medical University of Silesia, Katowice, Poland \\ ${ }^{2}$ Department of Radiology and Radiodiagnostics, Medical University of Silesia, Katowice, Poland
}

\section{Abstract}

\begin{abstract}
Purpose: Sacrococcygeal teratomas (SCT) can be detected in ultrasonography as early as in the first trimester. Currently, prenatal ultrasonography enables a thorough examination of tumors, but it is not always sufficient. The purpose of this study was to determine the most important features of SCTs in fetal magnetic resonance imaging and to confront them with postnatal computed tomography $(\mathrm{CT})$.
\end{abstract}

\begin{abstract}
Case report: Between 2009 and 2013, 5 cases of sacrococcygeal teratomas were diagnosed in our hospital using fetal magnetic resonance imaging ( 3 female and 2 male infants). Three of the affected newborns underwent postnatal CT before surgery. In each case, tumor size, its content, mass effect, and classification according to the Altman's criteria were determined and compared with other features. Fetal magnetic resonance imaging (MRI) and postnatal CT were in excellent agreement with respect to tumor classification using the aformentioned criteria. MRI better characterizes tumor content and its extent compared to ultrasound, and enables a precise structural assessment of the central nervous system. Postnatal CT is complementary to fetal MRI and optional.
\end{abstract}

Conclusions: Fetal MRI may help in the prenatal diagnosis of SCTs as it overcomes the limitations of obstetric ultrasound. Postnatal computed tomography is useful in determining tumor vascularity or calcifications, and it can depict the surrounding bone structures.

Key words: magnetic resonance imaging, multidetector computed tomography, sacrococcygeal region, teratoma.

\section{Introduction}

Of all congenital tumors, sacrococcygeal teratoma (SCT) is the most common, with a reported incidence of $1: 35,000-40,000[1,2]$ and a $3: 1$ female predominance [3]. Recent studies suggest that the incidence can be as high as $1: 14,000$ [3], and prenatally diagnosed tumors account for $50 \%$ of cases [4]. Fetal teratomas are believed to arise from an aggregation of totipotent cells in the primitive streak, also called the Hensen's node. Remnants of this area may persist and give rise to SCTs that contain cells from all 3 germ layers. While the majority of these tumors are benign, perinatal mortality rates are high and range from $13 \%$ to $16 \%$ for prenatally diagnosed cases [4]. These perinatal deaths are caused mostly by preterm delivery, cardiac failure, or damage to the tumor resulting in hemorrhage $[5,6]$. Large teratomas are highly vascular and contain significant amounts of blood which may lead to fetal anemia and high-output cardiac failure [3,5]. The clinical course of fetal SCTs is often unpredictable, with some tumors growing rapidly while others retain their initial size. Numerous predictive factors have been

Correspondence address:

Jolanta Myga-Porosiło, Department of Radiology and Radiodiagnostics, Medical University of Silesia, Medyków 18 Str., 40-055 Katowice, Poland,

e-mail:jmyga@interia.pl

Authors' contribution:

A Study design · B Data collection · C Statistical analysis · D Data interpretation · E Manuscript preparation · F Literature search · G Funds collection 
Table 1. Sacrococcygeal teratoma staging according to the American Academy of Pediatrics Surgery Section Survey

\begin{tabular}{|l|l|}
\hline Tumor type & Description \\
\hline I & The tumor is developing in its entirety outside the fetus \\
\hline II & The tumor has an internal, intrapelvic component in addition to the external part \\
\hline III & The majority of the tumor is located intrapelvically or inside the abdomen, with only a small external component \\
\hline IV & The entirety of the tumor is located inside the fetus \\
\hline
\end{tabular}

investigated, including tumor weight to fetal volume ratio, tumor structure, and vascularity [3,6-8]. In this study, we describe magnetic resonance (MRI) and computed tomography (CT) features of SCTs in 5 newborns, and discuss the benefits and limitations of these imaging modalities.

\section{Material and methods}

Between 2009 and 2013, 5 cases of SCTs were diagnosed in our center ( 2 males, and 3 females). The patients were referred for outpatient magnetic resonance imaging (MRI) after a diagnosis of SCT was made during routine prenatal ultrasound screening. The indications in all of these cases were clinical picture, medical history, and ultrasound results. In one case, a subsequent prenatal MRI was performed due to a fast growth rate of the tumor. In 3 newborns, postnatal CT was performed shortly before surgery to assess tumor extension, size, associated vessels,

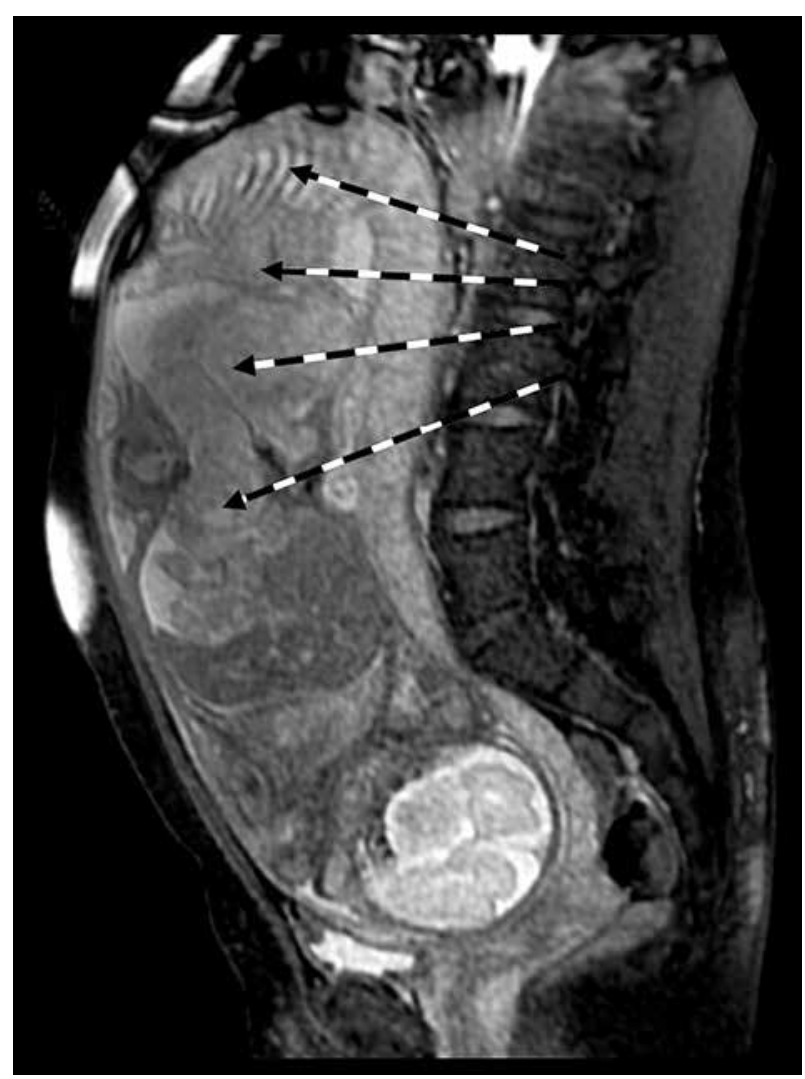

Figure 1. Appearance of type III sacrococcygeal teratoma in magnetic resonance imaging. The arrows indicate the extent of the tumor. and origin, as ordered by pediatric surgeons. The mean gestational age at the time of the first MRI examination was 26 weeks. The imaging was performed using a $1.5 \mathrm{~T}$ General Electric's HDx device using FSPGR T1fs, SSFSE T2, and 2D FIESTA sequences in transverse, sagittal, and frontal planes. Layer thickness of $5 \mathrm{~mm}$ and $3 \mathrm{~mm}$ was used. The platform was the property of the Voxel Medical Diagnostics Center. Proper permissions were given by the company for the use of their diagnostic images in this article. When possible, the MRI were compared with ultrasound examinations. CT of the abdomen and lesser pelvis was performed in 3 newborns because of surgical indications, using multi-row polyphasic spiral technique. These scans were performed shortly before surgery in the Department of Radiology, as ordered by pediatric surgeons. In some cases, contrast was administered intravenously. Layer thickness was $1.25 \mathrm{~mm}$, and delayed phase was registered after 10 minutes. Scans were acquired with the Toshiba Aquilion 16 CT platform. In every case, tumor size, its extent, and mass effect on nearby tissues were evaluated. MR and CT images were compared with other postnatal findings, i.e. a histopathological examination. Every tumor was classified according to the Altman's criteria (American Academy of Pediatrics Surgery Section Survey) (Table 1).

\section{Results}

In 4 of the reviewed cases, type I SCT was diagnosed, and type III tumor was seen in 1 patient.

\section{Type III sacrococcygeal teratoma}

A 28-year-old primagravida was referred for an outpatient MRI after a diagnosis of presacral tumor was made on a routine ultrasound examination. MRI was performed in the $30^{\text {th }}$ week of pregnancy. The scan revealed a large $(14 \times 10 \times 15 \mathrm{~cm})$, polycyclic, heterogenic mass located in the fetal pelvis that adhered to the coccyx. An abdominal extension was observed (Figure 1), and the teratoma was classified as type III. The tumor was of a solid-cystic structure with some liquid components. The pyelocalyceal system was dilated, and the bladder was displaced upwards by the tumor. The intestines appeared irregular due to displacement by the tumor. No abnormalities were noted in the central nervous system. The extremities of the fetus were difficult to assess because of their position and tightness caused by the tumor. Due to a fast rate of 
growth and its large size, cesarean section was performed at $34 \mathrm{Hbd}$. The newborn was referred by pediatric surgeons for preoperative CT. CT revealed a large, solid-cystic tumor with heterogeneous calcifications located in the pelvis with an abdominal extension and an external component. The findings were in line with MRI. However, tumor vascularity was assessed more precisely (Figure 2). Tumor vessels originated from the internal iliac artery, with additional branches originating from external iliac arteries, inferior mesenteric artery and median sacral artery. The tumor was also supplied with blood through partially formed vessels of the dorsal muscles, especially in the sacral area. The newborn underwent successful surgery with good recovery. Unfortunately, 9 months later its condition deteriorated and the child was admitted to the ICU. Ultrasound and CT revealed a tumor located in the liver, with multiple metastases. Based on a histopathological examination, a diagnosis of hepatoblastoma was made.

\section{Type I sacrococcygeal teratoma}

The second case was seen in a male newborn. The pregnancy was terminated through cesarean section after 30 weeks of gestation due to a fast growth of the tumor and its large size $(7.7 \times 9 \times 13 \mathrm{~cm})$, which was revealed on ultrasound and MRI. The tumor, classified as type I, ruptured during the cesarean section causing a profuse bleeding. Non-enhanced CT was performed before surgery, and revealed a heterogeneous mass with numerous calcifications and a $3 \times 2.5 \mathrm{~cm}$ rupture in the tumor (Figure 3). The newborn died of blood loss and subsequent heart failure shortly before surgery.

The third case was seen in a female newborn with type I SCT. The first MR scan was performed in the $26^{\text {th }}$ week of pregnancy, and revealed a heterogeneous tumor, $9.5 \times$ $9.5 \times 6 \mathrm{~cm}$. As in the first case, the tumor consisted of solid and cystic elements, including liquid components. A proper assessment of the fetal extremities was impossible due to the mass of the tumor. CT findings were in line with MRI.

The fourth case was observed in a female newborn with a tumor measuring $3 \times 3.6 \times 2.4 \mathrm{~cm}$. Similarly to previous cases, assessment of the fetal extremities was difficult due to movement artifacts. CT findings were in line with MRI.

The fifth case was observed in a male newborn with a type I teratoma measuring $21 \times 13.2 \times 9.3 \mathrm{~cm}$. MRI excluded any internal component. A solid-cystic structure of the tumor was of a heterogeneous character and included many calcifications. CT findings were in line with MRI, and revealed a complex network of vessels originating from the right internal iliac artery.

\section{Discussion}

With recent studies $[3,9,10]$ reporting a higher incidence of SCTs compared to earlier reports, diagnostic problems posed by these tumors may be more common than previ-

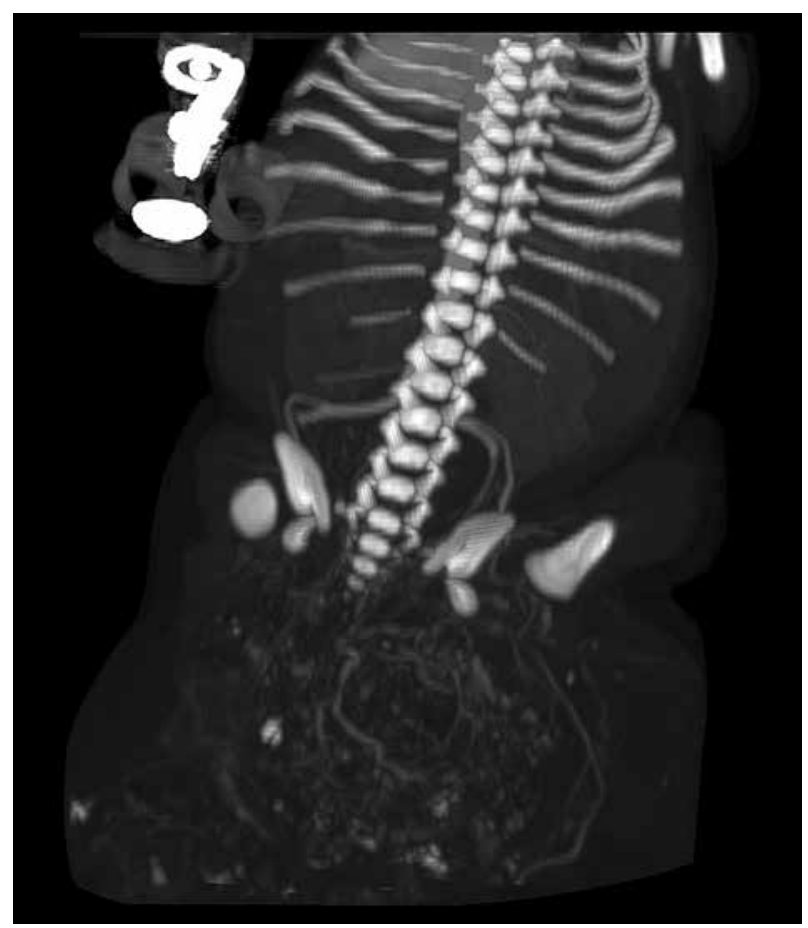

Figure 2. The tumor's vessels visible in contrast computed tomography after birth

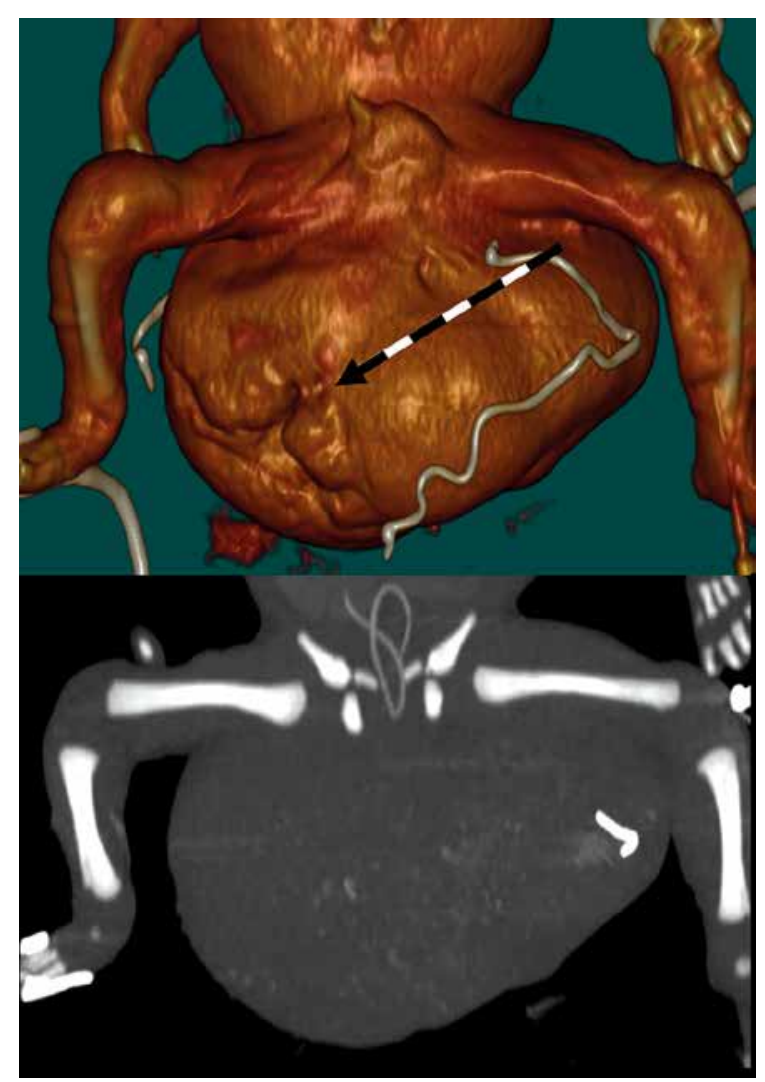

Figure 3. Computed tomography of sacrococcygeal teratoma. Surface 3D rendering reveals the tumor rupture that occurred during the cesarean section (indicated by the arrow)

ously believed. Prenatally diagnosed SCTs carry a higher risk of preterm delivery [1] and have a high mortality rate of $13-16 \%$ [4]. Therefore, these pregnancies should be monitored more carefully using complementary diagnos- 


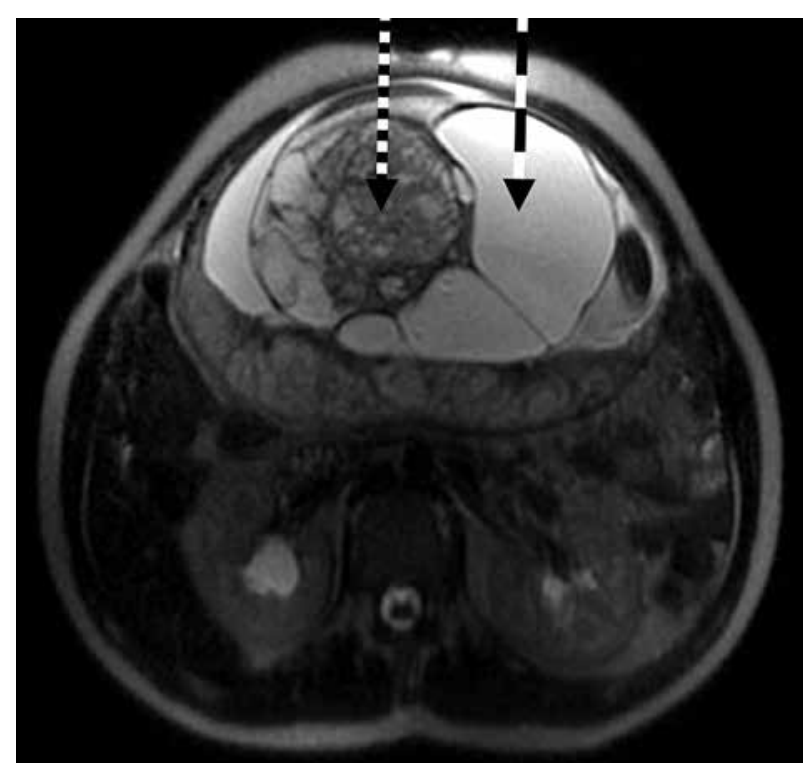

Figure 4. Mixed (left arrow) and liquid (right arrow) components of a sacrococcygeal teratoma in fetal magnetic resonance imaging

tic techniques when needed, such as magnetic resonance imaging. Prenatal imaging yields useful information for obstetricians, gynecologists, and surgical teams. Useful tumor features that can assessed in imaging include tumor size, morphology, vascularity, growth rate, and tumor volume to fetal weight ratio (TFR) [14]. These features can serve as prognostic factors which may help determine further management [3]. Ultrasonography remains the most important imaging modality for routine screening during pregnancy [11]. It is a widely available, cost-efficient, and safe method that allows a real-time examination of the fetus [12]. When performed serially, obstetric ultrasound is also a simple way of monitoring the dynamics of SCT growth, and can reveal tumor content [13]. Tumor growth rates of more than $150 \mathrm{~cm}^{3}$ per week have been associated with increased perinatal mortality [15]. However, ultrasound has some limitations as an imaging modality that can be addressed by magnetic resonance imaging or postnatal computed tomography. The limitations of ultrasound include a limited field of view and difficulties in penetrating bones which results in acoustic shadowing. In addi Therefore tion, ultrasound assessments may be limited by the fetus position, maternal obesity, oligohydramnios, and other problems [13]. In prenatally diagnosed SCT cases, fetal magnetic resonance should be considered as a complementary imaging modality if the ultrasound examination is uncertain, could not be completed, or a more precise assessment is needed, i.e., if the tumor has a large intra-pelvic or abdominal component. On MRI scans, SCTs appear as heterogenic, presacral masses of varying extent. The tumor usually consists of tissue, fat, and liquid components in varying proportions (Figure 4).

Each of these components can be identified in MRI based on signal intensity. The tissue component is characterized by intermediate signal intensity in both T1- and T2-dependent sequences. Fat is highly hyperintense in

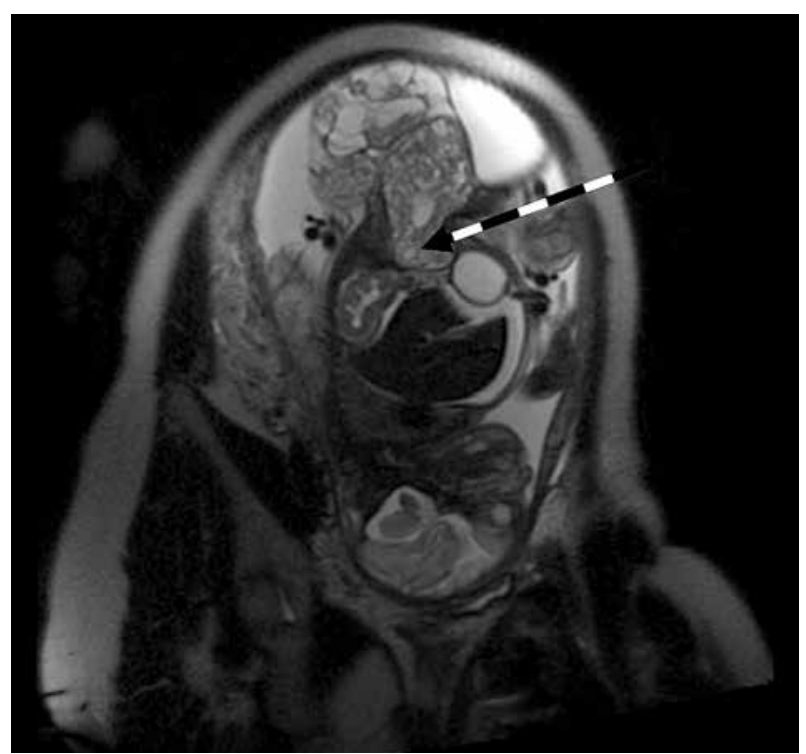

Figure 5. Appearance of sacrococcygeal teratoma in magnetic resonance imaging, $30 \mathrm{Hbd}$. The arrow indicates the internal component of the tumor

$\mathrm{T} 1$ and T2 sequences, and is suppressed in fat-saturation sequences. Liquid components are T2-hyperintense and T1-hypointense. It is possible to identify hemorrhagic changes associated with the tumor using MRI [16]. MRI enables a precise classification of the extent of the tumor using the Altman's criteria. In the reviewed cases, there was a complete agreement between MRI and CT with respect to tumor classification using the AAPSSS scale (Table 1). Unfortunately, MRI has significant limitations as well. These include movement artifacts created by the fetus, lack of contrast enhancement that significantly affects the evaluation of tumor blood vessels, and poor imaging of bone structures and calcifications. Krekora et al. reported a patient in whom right forearm aplasia and absence of hand were not detected on fetal ultrasound and MRI [13]. Such problems may be due not only to movement artifacts but also to tightness caused by large tumors (Figure 5).

In patients reviewed in our article, proper assessment of the fetal extremities was often difficult due to the tumor mass and movement artifacts. However, MRI remains the method of choice for assessing the fetal central nervous system and enables differential diagnosis with disorders such as myelomeningocele or spina bifida.

\section{Conclusions}

Fetal MRI may help in the prenatal diagnosis of SCTs as it overcomes the limitations of obstetric ultrasound. Postnatal CT is useful in determining tumor vascularity, its calcifications, and it can depict the surrounding bone structures. Both techniques are valuable in diagnosing teratomas of the sacrococcygeal region.

\section{Disclosure}

The authors declare no conflict of interests. 


\section{References}

1. Avni FE, Guibaud L, Robert Y, et al. MR imaging of sacrococcygeal teratoma: Diagnosis and assessment. Am J Roentgenol 2002; 178: 179-183.

2. Kwiatkowski S, Zapałowska M, Mikołajek-Bedner W, et al. Fetal sacrococcygeal teratoma - a case report and literature overview. Perinatologia, Neonatologia i Ginekologia 2014; 7: 48-51 [in Polish].

3. Hambreus M, Arnbjörnsson E, Börjesson A, et al. Sacrococcygeal teratoma. A population-based study of incidence and prenatal prognostic factors. J Pediatr Surg 2016; 51: 481-485.

4. Swamy R, Embleton N, Hale J. Sacrococcygeal teratoma over two decades: Birth prevalence, prenatal diagnosis and clinical outcomes. Prenat Diagn 2008; 28: 1048-1051.

5. Kremer MEB, Wellens LM, Derikx JPM, et al. Hemorrhage is the most common cause of neonatal mortality in patients with sacrococcygeal teratoma. J Pediatr Surg 2016; 51: 1826-1829.

6. Akinkuotu AC, Coleman A, Shue E, et al. Predictors of poor diagnosis in prenatally diagnosed sacrococcygeal teratoma: A multi-institutional review. J Pediatr Surg 2015; 50: 771-774.

7. Coleman A, Shaaban A, Keswani S, Lim FY. Sacrococcygeal teratoma growth rates predict adverse outcomes. J Pediatr Surg 2014; 49: 985-989.

8. Altman RP, Randolph JG, Lilly JR. Sacrococcygeal teratoma: American Academy of Pediatrics Surgical Section survey. J Pediatr Surg 1974; 9: 389-398.
9. Pauniaho SL, Heikinheimo O, Vettenranta K, et al. High prevalence of sacrococcygeal teratoma in Finland - a nationwide population-based study. Acta Paediatr 2013; 102: 251-256.

10. Derikx JP, De Backer A, van de Schoot L, et al. Long-term functional sequelae of sacrococcygeal teratoma: A national study in the Netherlands. J Pediatr Surg 2007; 42: 1122-1126.

11. American College of Obstetricians and Gynecologists: Ultrasound in pregnancy. Practice Bulletin No. 175. Obstet Gynecol 2016; 128: 241-256.

12. Blaicher W, Prayer D, Bernaschek G. Magnetic resonance imaging and ultrasound in the assessment of the fetal central nervous system. J Perinat Med 2003; 31: 459-468.

13. Krekora M, Zych-Krekora K, Blitek M, et al. Difficulties in prenatal diagnosis of tumour in the fetal sacrococcygeal area. Ultrasound 2016; 24: 119-124.

14. Rodriguez MA, Darrell LC, Lazar DA, et al. Tumor volume to fetal weight ratio as an early prognostic classification for fetal sacrococcygeal teratoma. J Pediatr Surg 2011; 46: 1182-1185.

15. Wilson RD, Hedrick H, Flake AW, et al. Sacrococcygeal teratomas: Prenatal surveillance, growth and pregnancy outcome. Fetal Diagn Ther 2009; 25: 15-20.

16. Danzer E, Hubbard AM, Hedrick HL, et al. Diagnosis and characterization of fetal sacrococcygeal teratoma with prenatal MRI. Am J Roentgenol 2006; 187: 350-356. 